\title{
Analysis of seven face-on spiral galaxies ${ }^{\star}$
}

\author{
E. Pompei ${ }^{2}$ and G. Natali ${ }^{1}$ \\ 1 Istituto di Astrofisica Spaziale C.N.R.-I.A.S. Via Enrico Fermi 23, 00044 Frascati (RM), Italy \\ e-mail: natali@vaxtov.roma2.infn.it \\ ${ }^{2}$ Now at: Università di Trieste, Dip. di Astronomia, S.I.S.S.A.e Via Beirut 2-4 34013 Trieste, Italy \\ e-mail:pompei@newton.sissa.it
}

Received July 16; accepted November 16, 1996

\begin{abstract}
We continue in this work the study of nearby spiral galaxies started with NGC 628 (Natali 1992, hereafter Paper I). A sample of seven face-on spiral galaxies has been selected to obtain images in the $U, B, V$, $R, I$ bands using a CCD detector at the $90 \mathrm{~cm}$ Schmidt telescope at Campo Imperatore Observatory. Surface photometry using a dedicated software is used to derive both the surface brightness radial profiles in each band and the colour indexes. The radial disk scalelengths obtained from the radial profiles show a trend with wavelength which seems to highlight a huge presence of dust in the inner disk zone. For two galaxies of the sample the presence of dust is confirmed by the radial trend of colour indexes. The presence of galactic infall already detected for NGC 628 , is confirmed, from the bluish $(B-V)$, also for NGC 3184.
\end{abstract}

Key words: galaxies, spiral — photometry

\section{Introduction}

A significant quantity of photometric data on spiral galaxies is today available in the literature, but, very often, only measurements in one or two photometric bands (see De Vaucouleurs et al., RC3) are performed, while it would be more useful to have multiband photometry at disposal. From measurements in all the five photometric bands of the Johnson and Kron extended system, it is possible to obtain information on the dust distribution, on the various kinds of stellar populations present, and on their distribution throughout the galaxy. Data obtained in the $U$ and $B$ bands are very dust-sensitive and highlight the emission coming from ionized gas regions and from young stars; data from the $V$ band bring out mainly the emission coming from A-type dwarfs, while the $R$ and $I$ bands are more sensitive to the old stellar population, like that of K-type

^ Send offprint request to: E. Pompei giants. Moreover, these last two bands are less sensitive than the previous ones to the presence of dust. The study of the surface brightness radial profiles of galaxies allows to distinguish the zones of recent star formation; according to Boroson (Boroson 1981), in fact, when star formation is limited to certain regions of the disk, a departure from an exponential disk profile occurs. This interpretation seems confirmed by the work by Prieto et al. (1992). In their work the authors find that the non exponential behaviour in the bulge to disk transition zone (Freeman II profile) can be ascribed to a young stellar population hidden by dust. According to this work, the presence of dust can be enlightened by reddening and dimming in the light coming from the galactic zone considered. The study of the variation of physical parameters, like the bulge and disk scalelengths, versus wavelength, can help the comprehension of star formation histories of the galaxies. In a recent work, Ryder \& Dopita (1994, hereafter RD), have found that, for a sample of 34 spiral galaxy, the $V$ scalelength is longer than the I one. This is due to the fact that each new stellar generation adds a contribution to the scalelength, instead of following the pattern of the old one. On the contrary, if the dust contribution is underestimated, and the Holmerg approximation (Holmberg 1958) is wrong, (i.e. there is much more dust inside the disk), the behaviour of the disk scalelength is dependent on the adopted dust model (see Disney et al. 1989, hereafter DDP). The study of the trend of colour indexes with radius has been used to give an experimental confirmation (see Paper I) to the infall model of Larson \& Tinsley (Larson \& Tinsley 1978, herafter L\&T78). We have acquired, for a sample of seven spiral galaxies, photometric data in five photometric bands $U, B, V, R, I$ of the Johnson and Kron extended system, and we have analyzed the surface brightness radial profiles of the galaxies and the radial trend of colour indexes $(B-V),(B-I),(U-B)$. In the following section we describe the observations, while in Sect. 3 we present the data reduction and analysis with some comments on each galaxy; finally in Sect. 4 we discuss the results obtained. 

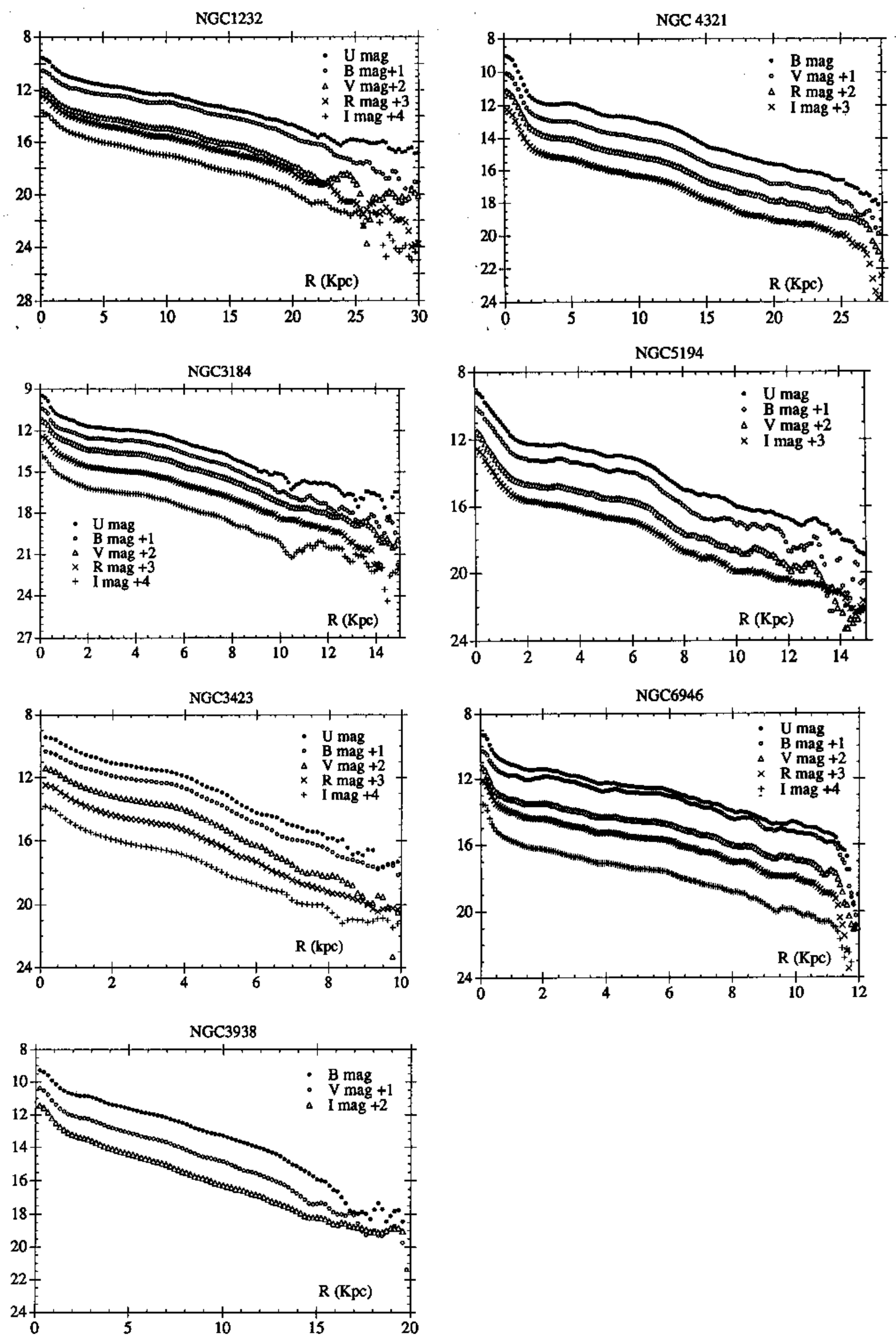

Fig. 1. Photometric profiles in $U B V R I$ bands for all galaxies. The magnitudes have been rescaled by the indicated quantities for clarity's sake 


\section{Observations}

For the measurements we have used a CCD detector placed at the prime focus of the Schmidt telescope at Campo Imperatore (AQ) Observatory (Natali et al. 1990). The dimensions of the telescope are $(60 / 90 / 180) \mathrm{cm}$. The detector was equipped with a Thomson chip TH7882, front illuminated with a metachrome 2 coating to enhance its sensibility to the $U$ and $B$ photometric bands. The linear dimensions of the sensitive area are $(13.25 \times 8.83) \mathrm{mm}^{2}$, organized in $(384 \times 576)$ pixels $(23 \mu \mathrm{m} \times 23 \mu \mathrm{m})$, which are equivalent at the telescope focus, to a field of view of $25^{\prime} \times 17^{\prime}$ (scalefactor $\left.=2.64 \mathrm{arcsec} / \mathrm{pixel}\right)$. The sample has been selected according to the following criteria:

a) homogeneity: all objects have morphological types between Sbc and Scd, to avoid any dependence of the measured quantities on morphology;

b) small inclination angles $\left(i<30^{\circ}\right.$, except for NGC 6946, with $i=42^{\circ}$ ), to reduce autoabsorption effects;

c) angular diameters between $15^{\prime}$ and $3.3^{\prime}$, for a better use of the great field of view.

The objects are listed in Table 1; all the parameters listed in the table, except some values of the inclination angle, which are taken from the Uppsala General Catalog (when a number is used for the inclination angle), and the inclination angle of NGC $5194^{1}$, have been taken from the Nearby Galaxies Catalog. For each observing night, flatfield frames have been taken, but we haven't measured any standard stars, therefore the photometric magnitudes are differential.

Table 1. Objects observed

\begin{tabular}{|c|c|c|c|c|c|}
\hline Name & Type & $\begin{array}{r}D_{25} \\
\quad\left(^{\prime}\right)\end{array}$ & $B_{0, T}$ & ${ }^{i}$ & $\begin{array}{r}d \\
(\mathrm{Mpc})\end{array}$ \\
\hline NGC 1232 & $\mathrm{Sc}$ & 7.2 & 10.39 & 1 & 20 \\
\hline NGC 3184 & Scd & 7.5 & 10.37 & $26^{\circ}$ & 8.7 \\
\hline NGC 3423 & Scd & 4.0 & 11.50 & 1 & 10.9 \\
\hline NGC 3938 & $\mathrm{Sc}$ & 4.9 & 10.89 & $22^{\circ}$ & 17 \\
\hline NGC 4321 & $\mathrm{Sbc}$ & 6.1 & 10.00 & $1-2$ & 16.8 \\
\hline NGC 5194 & $\mathrm{SbcP}$ & 13.6 & 8.68 & $22^{\circ}$ & 7.7 \\
\hline NGC 6946 & Scd & 11.2 & 7.92 & $42^{\circ}$ & 5.5 \\
\hline
\end{tabular}

\section{Data analysis and reduction}

After bias and dark current subtraction, cosmic ray removal and flat field correction, we used, for frame preprocessing, a software package written at C.N.R. observing

1 ApJS 27, 347. station at Monte Porzio (RM). Gradients of background luminosity, due to imperfect flat field renormalization of non uniform sky brightness, were eliminated by subtracting the least square plane of the sky background image. The sky background in each band was estimated as the average of the median values of 15 aperture photometry measurements using $(25 \times 25)$ pxl. boxes located far from the galaxy. After this, all images were convoluted twice with a two pixels FWHM gaussian filter. The coordinates of each galaxy centre were calculated with a 0.2 pxl resolution, fitting a gaussian profile with the FWHM of the nucleus. As all galaxies, except NGC 6946, have small inclination angles, the radial surface brightness was derived by integration over circular rings centered on the galaxy. The luminosity of each ring, 1 pixel wide, was evaluated as follows: around the average brightness value it was considered an interval $10 \%$ of the average value wide. The surface brightness of the ring was the median of the pixels set in this interval (for more details, see Natali et al. 1992). For NGC 6946 some modifications were needed, to take into account the significative inclination angle. To derive the radial surface brightness for NGC 6946, we performed integration over elliptical rings, which are circular in the plane of the galaxy. The position of the rings was specified by the inclination and position angles, while the ring width and the luminosity calculations were the same as before. We obtained the radial surface brightness profile in the five photometric bands $U, B, V, R, I$, and the radial colour indexes $(B-V),(B-I),(U-B)$; we also calculated the radial scalelengths for the bulge and the disk. The scalelengths are defined in different ways by different authors, namely De Vaucouleurs (1974), Freeman (1970), Boroson (1981), Vila-Costas and Edmunds (1992), Kormendy (1977). In this work we have adopted the definitions of Vila-Costas and Edmunds for the bulge and of Freeman and Kormendy for the disk, so the equations are, for the disk:

$$
\begin{aligned}
& m(r)=m(0)+1.0857\left(\frac{r}{r_{\mathrm{d}}}\right) \\
& m(r)=m(0)+1.0857\left(\alpha r-\left(\frac{\beta}{r}\right)^{3}\right)
\end{aligned}
$$

and for the bulge:

$m(r)=m\left(r_{\mathrm{e}}\right)+8.327\left(\left(\frac{r}{r_{\mathrm{e}}}\right)^{0.25}-1\right)$

where:

$m\left(r_{\mathrm{e}}\right) \quad$ is the bulge half central magnitude

$m\left(r_{\mathrm{d}}\right)$ is $1 / \mathrm{e}$ the disk central magnitude

$m(0) \quad$ is the disk central magnitude

$r_{\mathrm{e}} \quad$ is the bulge scalelength

$r_{\mathrm{d}} \quad$ is the disk scalelength 
$\alpha, \beta \quad$ are the inverses of disk scalelengths.

We write the definition of the disk to bulge ratio as:

$D / B=0.28\left(\frac{r_{\mathrm{d}}}{r_{\mathrm{e}}}\right)^{2}\left(\frac{S_{\mathrm{d}}}{S_{\mathrm{e}}}\right)$

where:

$S_{\mathrm{d}} \quad$ is $1 / \mathrm{e}$ the disk central surface brightness

$S_{\mathrm{e}} \quad$ is $1 /$ e the bulge central surface brightness.

To obtain the disk and bulge scalelengths and the $D / B$ parameter, we fitted the disk using Eq. (1) or Eq. (2), obtaining $r_{\mathrm{d}}$; we subtracted the disk profile to the total one and, fitting the bulge with Eq. (3), we obtained $r_{\mathrm{e}}$. Finally we calculated $D / B$. The colour indexes were calculated by subtracting each other the relative magnitude values for each integration ring. In the following, discussion of the results obtained for each galaxy from data analysis is reported.

\section{NGC 1232:}

This galaxy has been classified as peculiar by H. Arp, because of the presence of a companion; its surface brightness radial profiles are presented in Fig. 1.

In Table 2 the values for the disk scalelengths for the five photometric bands, obtained using Eq. (1) are reported; in the second row there are the bulge scalelengths and in the third row the calculated values for the $D / B$ parameter. As can be seen from the table, the disk scalelengths decrease toward longer wavelengths; perhaps there is an increase in scalelength for each new stellar population (RD).

Table 2. $r_{\mathrm{d}}, r_{\mathrm{e}}, D / B$ for NGC 1232

\begin{tabular}{llllll}
\hline & $U$ & $B$ & $V$ & $R$ & $I$ \\
\hline$r_{\mathrm{d}}$ & 4.65 & 4.07 & 4.05 & 3.66 & 3.80 \\
$r_{\mathrm{e}}$ & 0.59 & 0.64 & 0.69 & 0.55 & 0.73 \\
$D / B$ & 8.24 & 7.02 & 3.11 & 7.63 & 3.86 \\
\hline
\end{tabular}

There is a good correspondence between our $D / B$ values and galaxy classification according van der Bergh, who assigns the subtype b to NGC 1232 (van der Bergh 1976). The colour indexes are given in Figs. $2 \mathrm{a}$ and $2 \mathrm{~b}$. For $(B-I)$ and $(B-V)$ there is a pronounced decreasing trend toward larger radii, with a shallower slope between 10 and $20 \mathrm{Kpc}$.

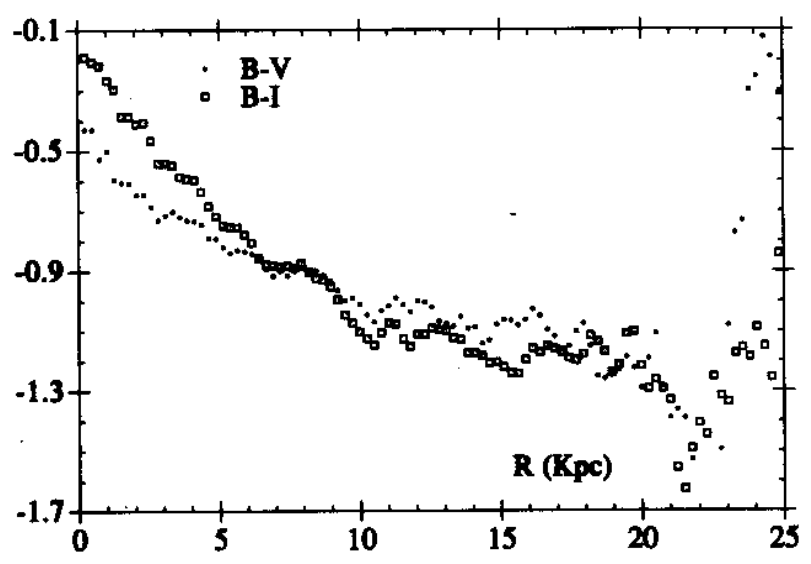

Fig. 2. a) Radial trend of the colour indexes $(B-V),(B-I)$ with galactocentric radius

It is plausible that the interaction with its companion has changed the matter distribution throughout the galaxy, determining the shallow trend in the colour indexes. Between 20 and $25 \mathrm{Kpc}$ there is a drop both in $(B-I)$ and in $(B-V)$, while the $(U-B)$ index shows an opposite trend, see Fig. $2 \mathrm{~b}$.

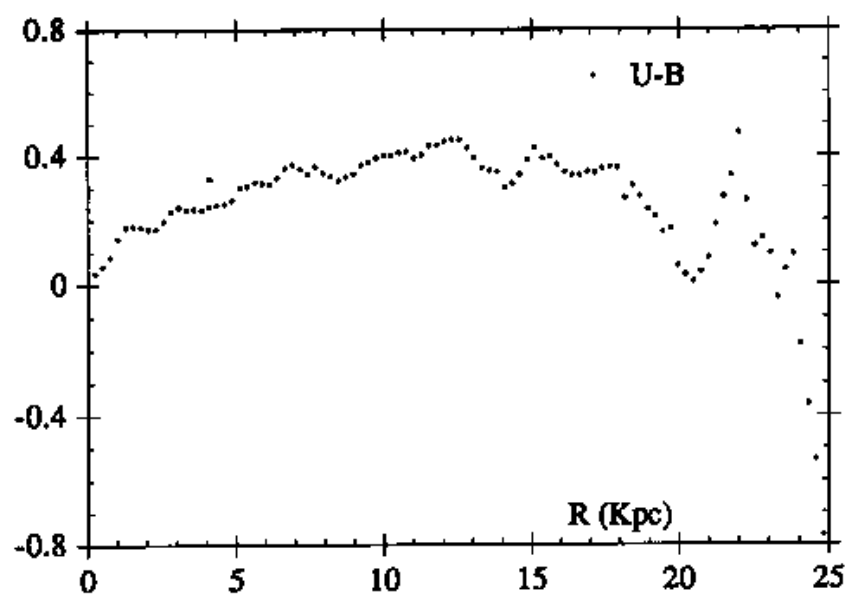

Fig. 2. b) Radial trend of the colour index $(U-B)$ with galactocentric radius

\section{NGC 3184:}

The radial surface brightness profiles are given in Fig. 1; as can be seen from the figure, there is a plateau between 2 and $4 \mathrm{Kpc}$, followed by the typical disk exponential behaviour. It is possible to classify these surface brightness profiles as Freeman II ones. The disk scalelengths, calculated according to Eq. (1), are given in the 
first row of Table 3 . The scalelengths in $B, V, R$ are almost costant, with a significative increase in the $I$ band; this behaviour supports the hypothesis of a significative amount of dust inside the galactic disk, because of the wavelength dependent absorption. However, the measurement in the $U$ band invalidates this hypothesis, if one doesn' $\mathrm{t}$ invoke the red-leak effect in the $U$ filter. From Fig. 3 one can see that the luminosity profile is almost completely dominated from the disk fit (1), $m(r)=m(0)+1.0857\left(\frac{r}{r_{\mathrm{d}}}\right)$, which implies the impossibility of calculating the bulge component and the scalelength except for the $I$ band, (see the second row of Table 3 ).

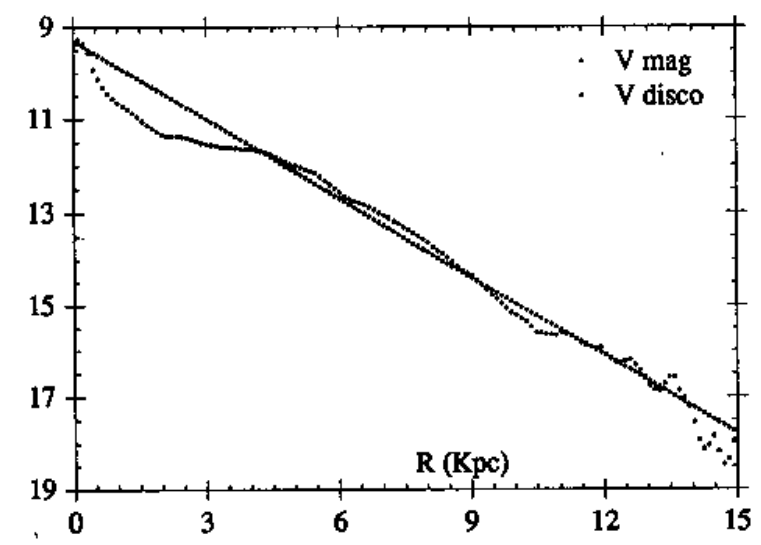

Fig. 3. Radial surface brightness profile in the $V$ band for NGC 3184 and, superimposed, the disk fit

The calculated $I$ bulge scalelength is $0.48 \mathrm{Kpc}$, with a $D / B$ parameter of 4.68 ; the resulting fit is given in Fig. 4 .

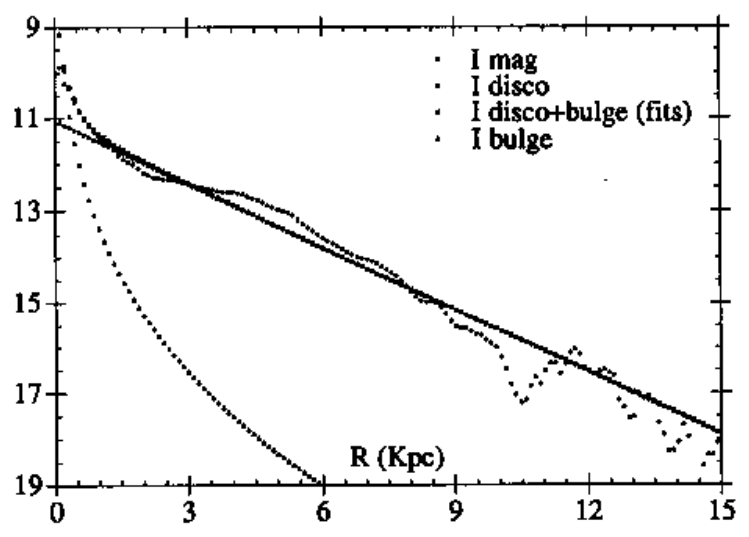

Fig. 4. Radial surface brightness profile in the $I$ band for NGC 3184. Superimposed, the disk fit, the bulge fit and the composite trend disk + bulge
Trying to obtain a better fit of the data, we have tried to fit the disk trend using Eq. (2),

$m(r)=m(0)+1.0857\left(\alpha r-\left(\frac{\beta}{r}\right)^{3}\right)$, with $\alpha=\beta$.

The results are given in Fig. 5 .

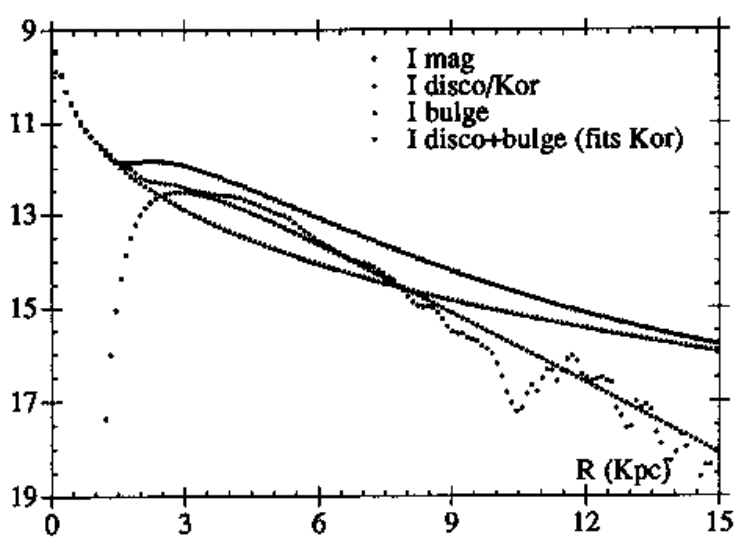

Fig. 5. As in Fig. 4, but the disk fit formula is given by Eq. (2)

As can be seen, the (2) and (3) give a good fit for the disk and bulge components separately, but the composite disk + bulge fit disagrees with data. The bulge scalelength obtained with this fit is very large, $10.17 \mathrm{Kpc}$ in the $V$ band, while the disk scalelength is $2.18 \mathrm{Kpc}$, with a $D / B$ parameter of 1 . The radial disk and bulge scalelengths obtained from (2) and (3) are given respectively in the third and fourth row of Table 3 . All the scalelengths are given in $\mathrm{Kpc}$.

Table 3. $r_{\mathrm{d}}, r_{\mathrm{e}}, D / B$ for NGC 3184

\begin{tabular}{lrrrrr}
\hline & $U$ & $B$ & $V$ & $R$ & $I$ \\
\hline$r_{\mathrm{d}}(1)$ & 2.22 & 1.82 & 1.92 & 1.942 .38 \\
$r_{\mathrm{e}}$ & - & - & - & -0.48 \\
$r_{\mathrm{d}}(2)$ & - & - & 2.18 & - & - \\
$r_{\mathrm{e}}$ & - & - & 10.17 & - & - \\
$D / B(1+3)$ & - & - & - & -4.68 \\
$D / B(2+3)$ & - & - & 1 & - & - \\
\hline
\end{tabular}

A comparison between Figs. 4 and 5 highlights the improved agreement between fits and data obtained using the fits (1) and (3) than that obtained with (2) and (3). The colour indexes are given in Figs. 6 b.

Both in the $(B-V)$ and $(B-I)$ there is a drop for small radii, which proves the existence of a galactic infall, according to Larson \& Tinsley (L\&T78); a similar 


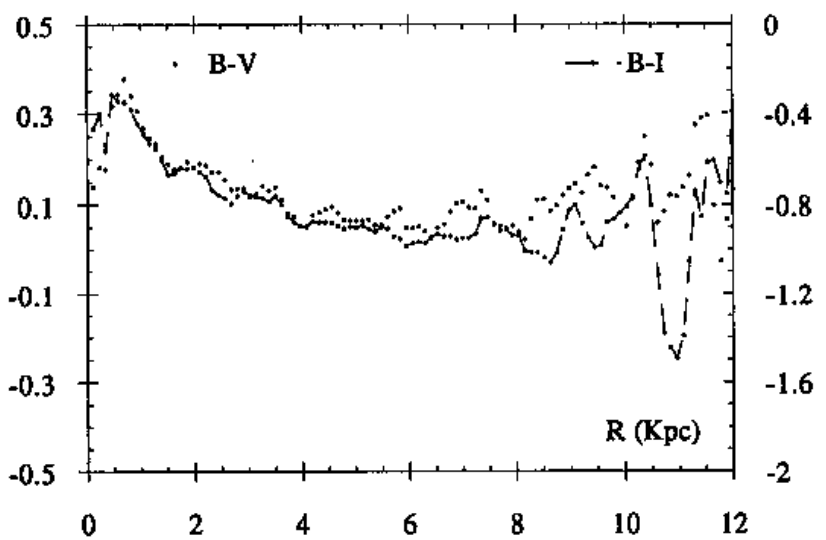

Fig. 6. a) Radial trend of the colour indexes $(B-V),(B-I)$ with galactocentric radius

behaviour has been detected in NGC 628 (see Paper I). There is also a pronounced depression between 10 and $12 \mathrm{Kpc}$ in $(B-I)$, while $(B-V)$ is smoother, but the noise does not allow to obtain a clear physical intepretation of this fact.

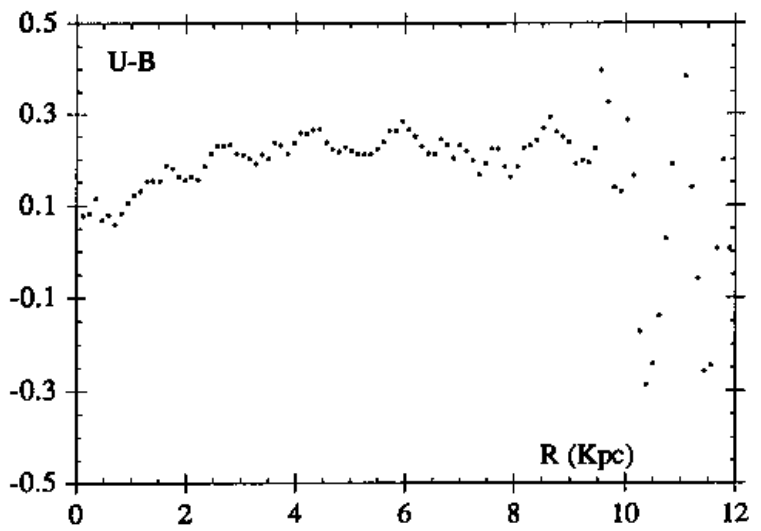

Fig. 6. b) Radial trend of the colour index $(U-B)$ with galactocentric radius

\section{NGC 3423:}

As can be seen from Fig. 1, the radial surface brightness profiles show a rapid change in slope at $3 \mathrm{Kpc}$, while between 0 and $3 \mathrm{Kpc}$ the bulge profile is poorly marked, with almost no fluctuations. The profiles show a trend similar to that of NGC 3184 in the bulge to disk transition zone, so we have attempted to fit the disk using Eq. (2). The results are better than those obtained in the former case, with a $I$ bulge scalelength of $1.15 \mathrm{Kpc}$, but there is a disagreement between the data and the composite fit disk + bulge for radii greater than $7 \mathrm{Kpc}$, (see Fig. 7).

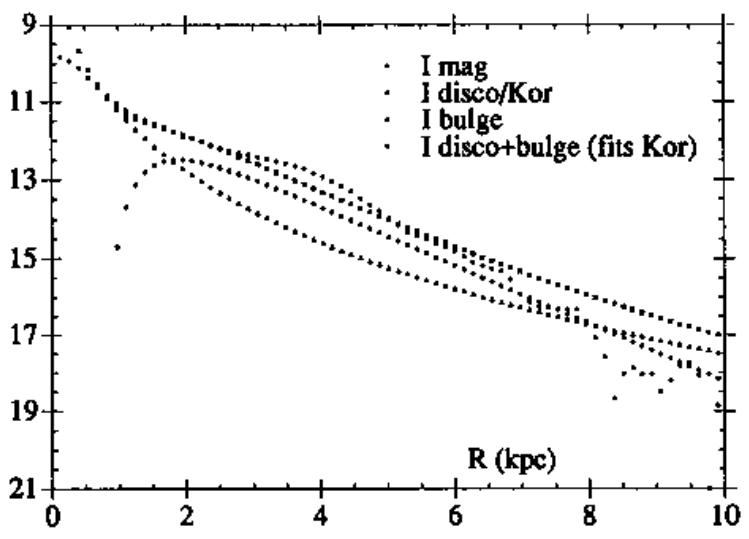

Fig. 7. Radial surface brightness profile in the $I$ band for NGC 3423. Superimposed, the disk fit, the bulge fit and the composite trend disk + bulge. The disk fit formula is given by Eq. (2), see text

The measured bulge scalelength is consistent with the galactic profile, but there is no plausible explanation for the disagreement between data and the fit, except that this deviation takes place in the outer zone, where the uncertainties in the data are larger. If Eq. (1) is used for the disk, the disk scalelengths in the first row of Table 4 are obtained, while the bulge and its scalelength can be measured only in the $I$ band. In the third and fourth rows of the same table are reported the $r_{\mathrm{d}}$ and $r_{\mathrm{e}}$ values obtained using Eq. (2) for the disk. Figure 8 shows the luminosity profiles fitted by Eq. (1) and Eq. (3); as it can be noted, there is a discrepancy between the total disk + bulge fit and the measured profiles. This discrepancy in the outer zone of the galactic disk is of the same order as that obtained using the Eq. (2) and Eq. (3), while the two fits are indistinguishable between 0 and $6 \mathrm{Kpc}$.

A useful constraint for choosing the fit may be the $D / B$ parameter; in fact, the latter must be large enough to justify the Tully clasification of this galaxy as Scd. The values obtained for $D / B$ are given in the fifth and sixth rows of Table 4 ; there is a dominant contribution from the bulge (which means small $D / B$ ) when the composite fit $(2)+(3)$ is used, while the value obtained when the $(1)+(3)$ fit is used, is the one corresponding to a late type galaxy, Sc, so we choose the Eq. (1) as the right fit for the disk.

A comparison between our disk scalelengths in the $V$ and $I$ bands with those obtained by Ryder \& Dopita (RD) shows that the disk scalelength we calculated has a bigger value in the $I$ band than in the $V$ one, while the opposite is true in their case; moreover the measured values are different: $2.1 \mathrm{Kpc}$ for the $V$ band and $1.9 \mathrm{Kpc}$ for the $I$ band, according to $\mathrm{RD}$, and $1.16 \mathrm{Kpc}$ for the $V$ band 


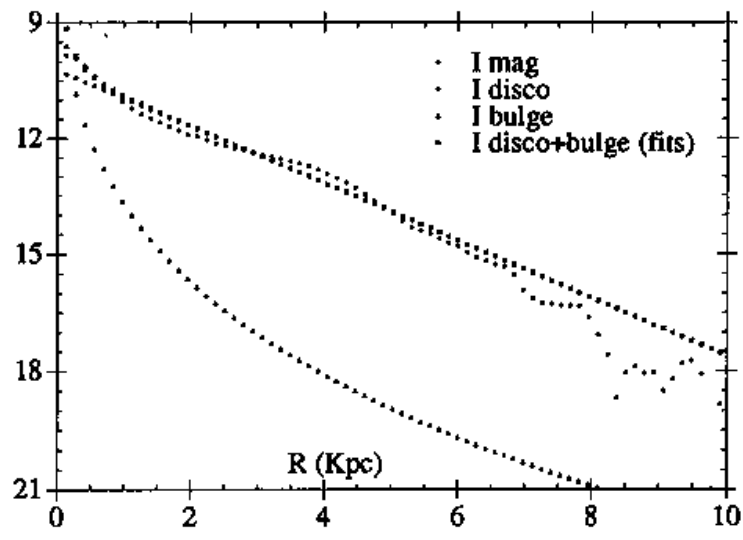

Fig. 8. As in Fig. 7, but the disk fit formula is given by (1)

Table 4. $r_{\mathrm{d}}, r_{\mathrm{e}}, D / B$ for NGC 3423

\begin{tabular}{llllll}
\hline & $U$ & $B$ & $V$ & $R$ & $I$ \\
\hline$r_{\mathrm{d}}(1)$ & 1.17 & 1.25 & 1.16 & 1.13 & 1.47 \\
$r_{\mathrm{e}}$ & - & - & - & - & 0.39 \\
$r_{\mathrm{d}}(2)$ & 1.31 & 1.50 & 1.38 & 1.41 & 1.42 \\
$r_{\mathrm{e}}$ & 0.77 & 1.11 & 1.02 & 1.02 & 1.15 \\
$D / B(1+3)$ & - & - & - & - & $>10$ \\
$D / B(2+3)$ & 2.40 & 1.48 & 1.66 & 1.76 & 1 \\
\hline
\end{tabular}

and $1.47 \mathrm{Kpc}$ for the $I$ band according to this work. This difference cannot be ascribed to a different choice of the galactic distance, because both we and RD have used the Tully catalog of nearby galaxies (Tully 1980). Our surface brightness profiles have a behaviour less smooth than those obtained by RD; moreover, their profiles seem to have a double slope in the disk zone, so, the discrepancy between the two disk scalelengths may arise from a different choice of the radial interval in which the exponential fit is applied. The scalelengths calculated from Eq. (2) have less internal dispersion. The colour indexes are given in Figs. 9b; it is possible to see a strong reddening in $(B-V)$ and $(B-I)$ toward small radii.

There is an interesting drop in both $(B-I)$ and $(B-V)$ between 5.5 and $6 \mathrm{Kpc}$, which may reveal a young stellar population in the outer edge of the galaxy.

The profiles are not really much significative for radii larger than $7-8 \mathrm{Kpc}$, because of the faintness of the object and the poor $\mathrm{S} / \mathrm{N}$.

\section{NGC 3938:}

For this object the surface brightness radial profiles were measured only in the $B, V, I$ bands (see Fig. 1) due

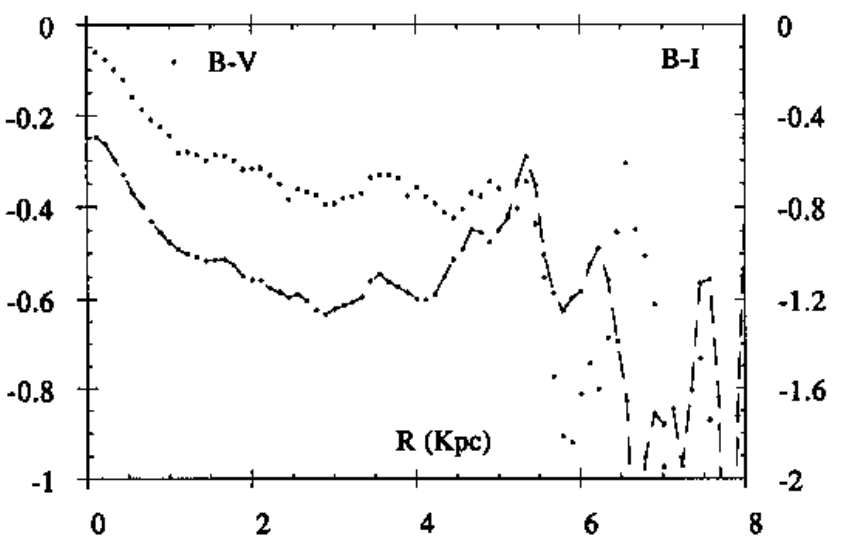

Fig. 9. a) Radial trend of the colour indexes $(B-V),(B-I)$ with galactocentric radius

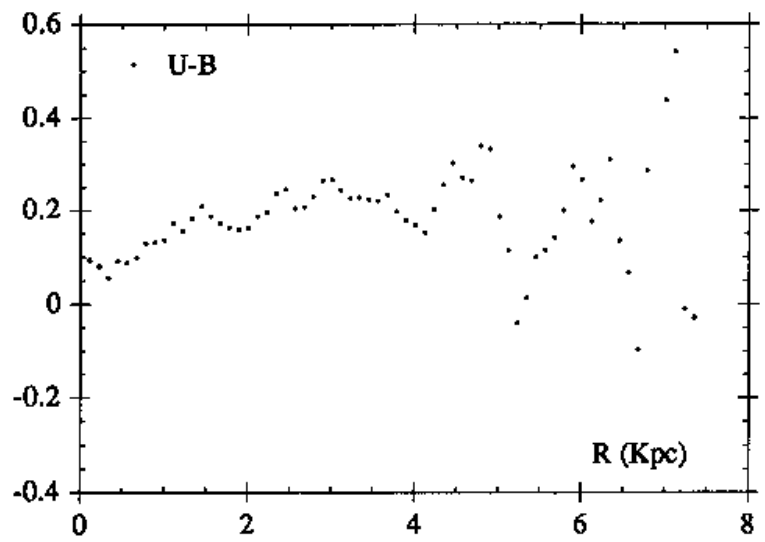

Fig. 9. b) Radial trend of the colour index $(U-B)$ with galactocentric radius

to bad weather conditions. In the $B$ band it has not been possible to calculate the bulge scalelength because of the disk domination in the inner zone of the galaxy. The disk and bulge scalelengths are given in Table 5 , together with the values of the $D / B$ parameter.

From the table, one can see that the larger variations in the disk scalelengths are in the $B$ and $V$ band; moreover, there is a decreasing trend in $r_{\mathrm{d}}$ toward shorter wavelengths when Eq. (1) is used, while the values are more or less constant when Eq. (2) is used. The great values obtained for the $D / B$ parameter give this galaxy a real late type classification, Sd, the Tully ones being Sc. The small bulge scalelengths in the $V$ and $I$ bands and the impossibility of measuring it in the $B$ band confirm the idea of a small bulge and then quite a big $D / B$. Figure 10 shows the $I$ band surface brightness radial profile and, superimposed to it, the composite fit disk plus bulge coming from the $(1)+(3)$; the colour indexes $(B-V)$ and $(B-I)$ are shown in Fig. 11.

As can be seen from Fig. 11, there is a pronounced reddening toward small radii for both indexes, the same as 
Table 5. $r_{\mathrm{d}}, r_{\mathrm{e}}, D / B$ for NGC 3938

\begin{tabular}{llll}
\hline & $B$ & $V$ & $I$ \\
\hline$r_{\mathrm{d}}(1)$ & 1.49 & 1.88 & 2.91 \\
$r_{\mathrm{e}}$ & - & - & 1.16 \\
$r_{\mathrm{d}}(2)$ & 3.16 & 2.91 & 3.04 \\
$r_{\mathrm{e}}$ & - & 0.61 & 1.05 \\
$D / B(1+3)$ & - & - & $>10$ \\
$D / B(2+3)$ & - & $>10$ & $>10$ \\
\hline
\end{tabular}

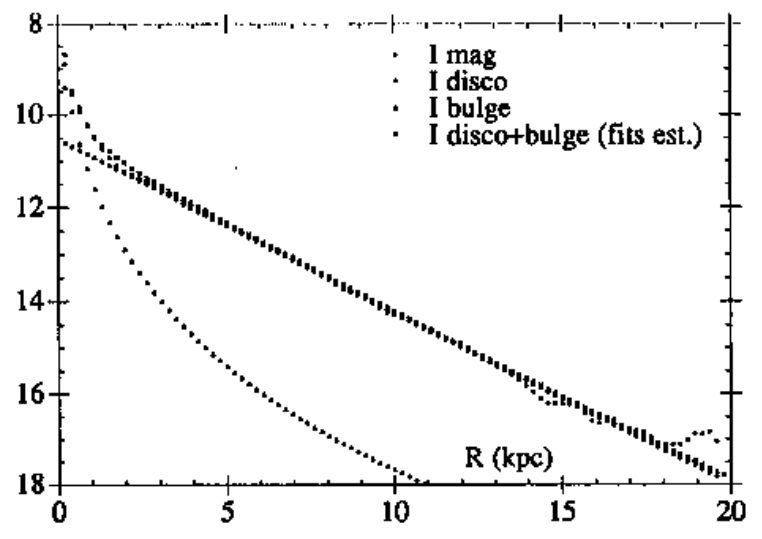

Fig. 10. The $I$ band surface brightness radial profile for NGC 3938 and, superimposed, the disk fit, the bulge one and the composite trend disk + bulge. The disk fit formula is given by Eq. (1), see the text

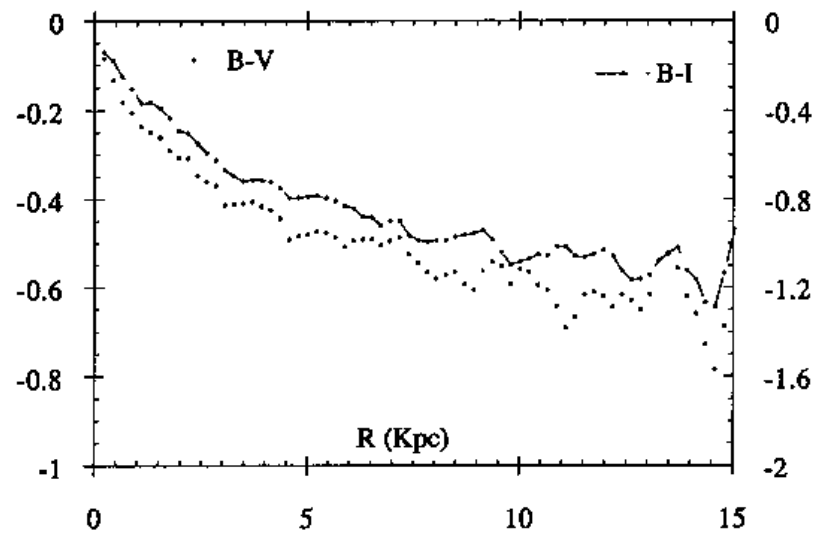

Fig. 11. Radial trend of the colour indexes $(B-V),(B-I)$ with galactocentric radius for NGC 3423. For bigger radii the indexes show a bluish trend, due to the existence of young stellar populations with different ages in the outer disk zone (L\&T78).

\section{NGC 6946:}

This galaxy has been extensively observed not only in the optical band, but also in the millimetric band, to study the $\mathrm{CO}$ regions by analyzing the rotational transition $J=1 \rightarrow 2$, at $2.6 \mathrm{~mm}$ (Young \& Scoville 1982), and in the radio band, to study the $\mathrm{HI}$ emision line. Photometric measures are difficult because of the great number of field stars, which must be eliminated before evaluating surface brightness profiles, and because of the large inclination angle. To obtain luminosity profiles, we have taken into account both of these factors, by eliminating field stars from the science frame and by integrating along ellipses in the observer's plane. The profiles show many bumps, with a sudden change in the disk slope at $6 \mathrm{Kpc}$, (see Fig. 1), and a drop in luminosity in the bulge-to-disk transition zone in the $U, B, V$ bands, which is not present in the $R$ and $I$ ones. There is another change in the disk slope at $11.5 \mathrm{Kpc}$, but it is not clear if it is due to an incomplete measure of galaxy luminosity or to a bad sky subtraction. The bulge and disk scalelengths, in Kpc, are given in Table 6 , together with the $D / B$ parameter, while in Fig. 12 the $B$ surface brightness radial profile is reported and, superimposed, the fit for the disk and the bulge.

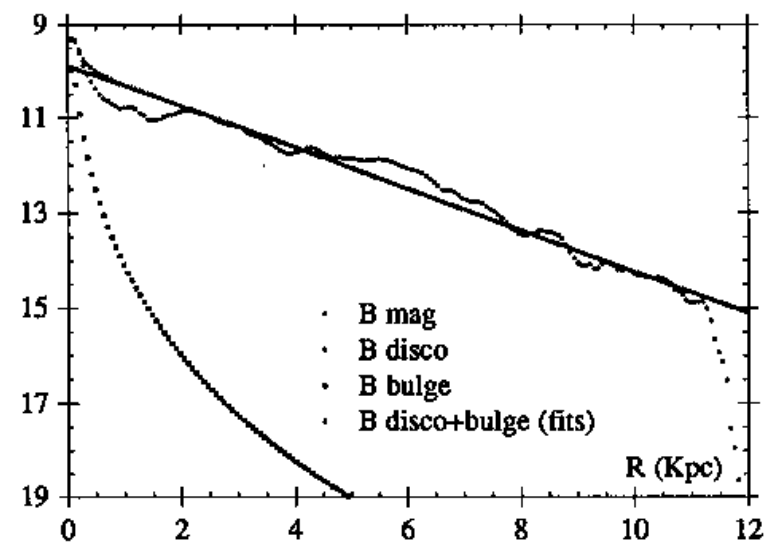

Fig. 12. The $B$ band surface brightness radial profile for NGC 6946 and, superimposed, the disk fit, the bulge fit and the composite trend disk + bulge. The disk fit formula is given by Eq. (1), see text

The scalelengths are in disagreement with those obtained from other authors, (see, for example, Young \& Scoville 1982), because the different choice of galactic distance, $10 \mathrm{Mpc}$ according Young and Scoville and $5.5 \mathrm{Mpc}$ according Tully (the value adopted in this work); 
Table 6. $r_{\mathrm{d}}, r_{\mathrm{e}}, D / B$ for NGC 6946

\begin{tabular}{llllll}
\hline & $U$ & $B$ & $V$ & $R$ & $I$ \\
\hline$r_{\mathrm{d}}(1)$ & 2.52 & 2.49 & 2.49 & 2.33 & 2.29 \\
$r_{\mathrm{e}}(1+3)$ & 0.40 & 0.50 & 0.48 & 0.52 & 0.33 \\
$D / B(10$ & $>10$ & $>10$ & $>10$ & $>10$ \\
$r_{\mathrm{d}}(7.5-11.5 \mathrm{Kpc})$ & 2.34 & 2.16 & 2.04 & 1.80 & 1.79 \\
\hline
\end{tabular}

the measurements of the luminosity profiles are indeed consistent with these two authors, including the $11.5 \mathrm{Kpc}$ knee. Disk scalelengths are shorter toward longer wavelengths, which means that each new stellar population makes the scalelength increase (see RD); on the contrary, if there were no variation of $r_{\mathrm{d}}$ with wavelength, each new stellar generation would follow the pattern established by the former ones. The values obtained for the $D / B$ parameter are in good agreement both with the galaxy classification by Tully and that from van der Bergh. Phillips et al. (Phillips et al. 1991), have hypothesized that this galaxy has an optically thick disk, and have corrected for autoabsorption using the DDP model and data from Young $\&$ Scoville. According to these authors, the disk becomes optically thin at $22-23 \mathrm{mag} B$, so we have attempted to fit the disk using its outer zone, $(7.5-11.5 \mathrm{Kpc}$; see Fig. 13), the profile is completely dominated by the disk. The disk scalelengths obtained using this radial interval are given in the last row of Table 6 ; as can be seen from the table, there is not much difference from those obtained using the whole disk, except in the $R$ and $I$ bands where the difference is $0.5 \mathrm{Kpc}$.

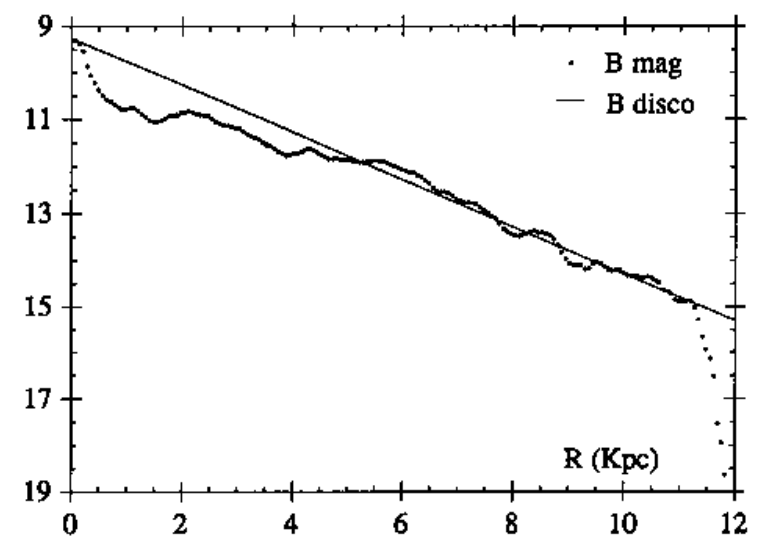

Fig. 13. The $B$ band surface brightness radial profile for NGC 6946 and, superimposed, the disk fit performed in the radial interval $7.5-11.5 \mathrm{Kpc}$. The disk fit formula is given by Eq. (1), see the text
This result, according to DDP, seems to confirm a huge presence of gas and dust inside the galaxy, already indicated by the luminosity drop in surface brightness radial profiles. The disk scalelengths shown in the last row of Table 6 have a decreasing trend toward longer wavelengths, that seems to confirm the DDP results, but it is in good agreement also with the RD work. The colour indexes are given in Figs. 14b.

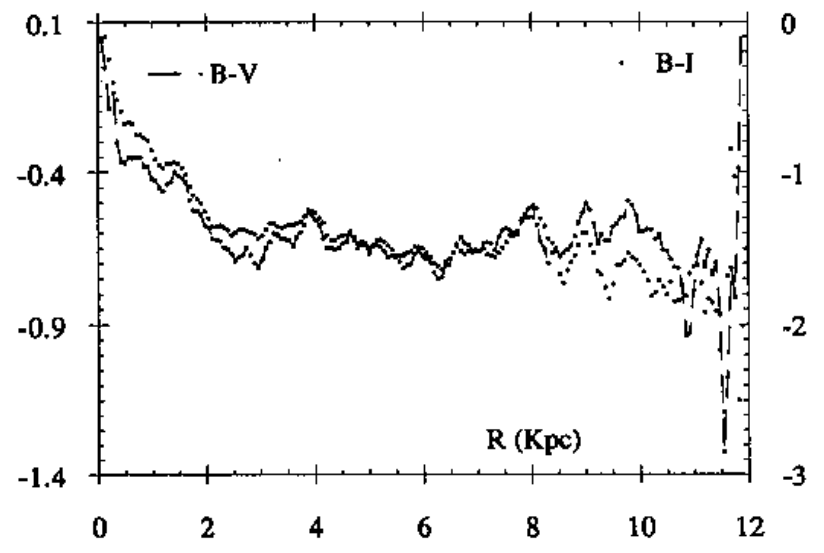

Fig. 14. a) Radial trend of the colour indexes $(B-I),(B-V)$ with galactocentric radius

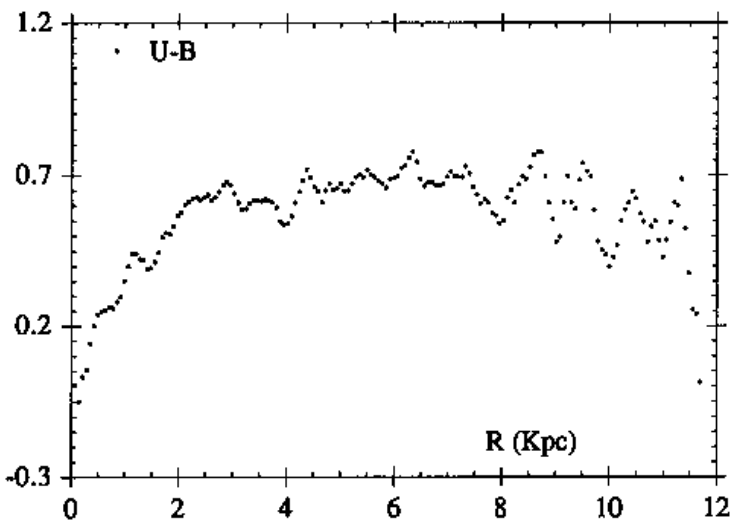

Fig. 14. b) Radial trend of the colour indexes $(U-B)$ with galactocentric radius

The indexes are superimposed for the whole radial range considered, and have a scaling ratio $1: 2$, while $(U-B)$ seems to have a specular behaviour with respect to the former indexes.

\section{NGC 4321:}

This is another peculiar galaxy, which is interacting with a companion. However, data analysis has not been 
modified to take into account the companion, so there is a non negligible influence of its presence in all photometric results, even if the surface brightness luminosity profiles in the $B, V, R, I$ bands (there are no $U$ band measurements due to technical problems during the observing night) are not very disturbed with respect to those of NGC 5194 (M 51), except for the presence of a drop in luminosity in the bulge to disk transition zone. This luminosity drop is more pronounced in the $B$ band than in the $I$ one, (see Fig. 1), as well as the luminosity fluctuation in the disk dominated zone of the profile. This is not unexpected, because according to numerical simulation models, (especially by Toomre 1972), the interaction with a companion perturbs the galaxy, changing its matter distribution and triggering a burst of star formation, which is detected mainly in the $B$ band. In the same band, the transitions between arm and interarm zones are more pronounced. In Table 7 the disk and bulge scalelengths are given, in $\mathrm{Kpc}$, together with the $D / B$ parameter.

Table 7. $r_{\mathrm{d}}, r_{\mathrm{e}}, D / B$ for NGC 4321

\begin{tabular}{lllll}
\hline & $B$ & $V$ & $R$ & $I$ \\
\hline$r_{\mathrm{d}}(1)$ & 4.42 & 4.46 & 4.47 & 4.51 \\
$r_{\mathrm{e}}$ & 1.78 & 1.83 & 1.84 & 1.85 \\
$D / B(1+3)$ & 4.16 & 4.64 & 4.16 & 3.19 \\
\hline
\end{tabular}

Both scalelengths and $D / B$ are consistent with the galaxy classification given by Tully, despite the interaction. There is a smooth trend of increasing disk scalelength toward the infrared, probably due to the presence of dust, which has a very patchy distribution due to the interaction. The colour indexes are given in Figs. 15b; they show a very irregular trend, with significative fluctuation also for small radii.

It is to be noted that the reddening in both indexes in correspondence of the luminosity drop in the surface brightness profiles is due to, (according to Prieto et al. 1992), the presence of a huge quantity of gas and dust. The presence of a bluish trend of the colour indexes toward small radii, $0-2.5 \mathrm{Kpc}$ seems to confirm the presence of galactic infall, (Larson 1976). The luminosity drop in the bulge to disk transition zone has led Phillips et al. (1991) to consider the disk of this galaxy as optically thick; however, as the galaxy is tidally disturbed and the same authors in a former work (DDP) state that interacting galaxies need a dedicated modellistic for dust distribution, we have not attempted to perform their analysis in this work.

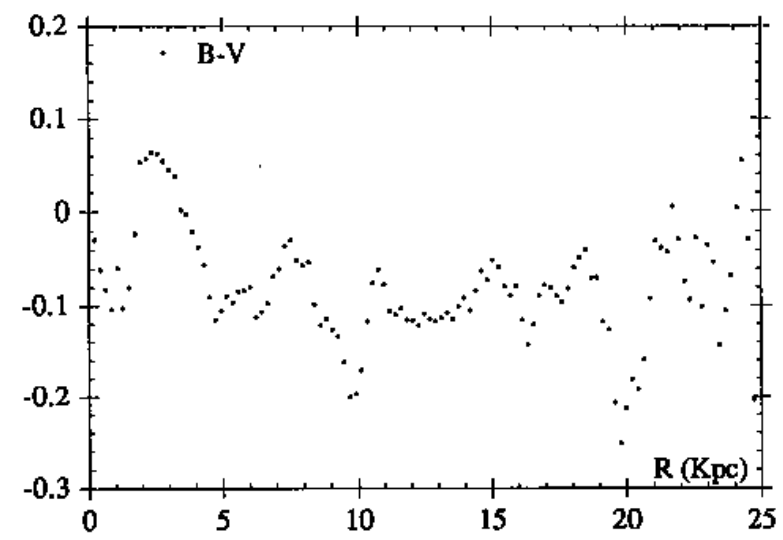

Fig. 15. a) Radial trend of the colour index $(B-V)$ with galactocentric radius

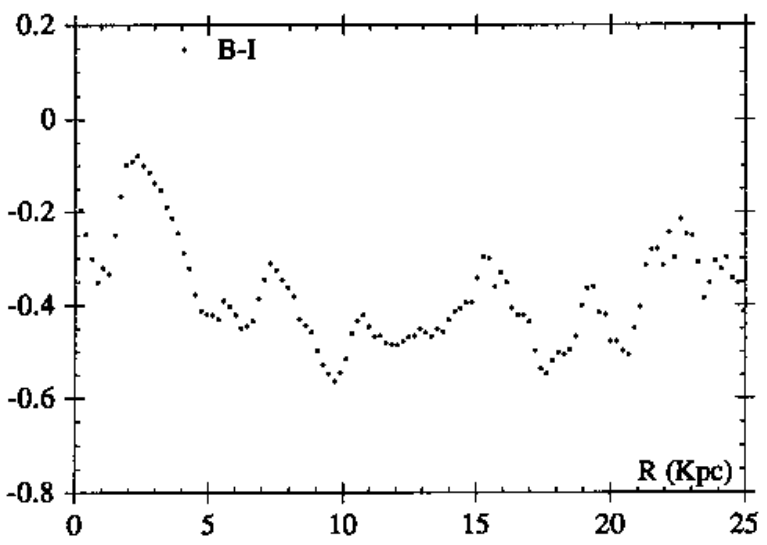

Fig. 15. b) Radial trend of the colour indexes $(B-I)$ with galactocentric radius

\section{NGC 5194:}

The third interacting galaxy is M 51. For this object we have slightly modified our data analysis, using circular sectors with an angular width of $135^{\circ}-435^{\circ}$, instead of circular rings, to avoid the luminosity contamination from the companion; in spite of this, the surface brightness radial profiles and the colour indexes have turned out to suffer from the presence of the interacting companion. The profiles show many bumps, (see Fig. 1), with a sudden change in the disk slope at $6.4 \mathrm{Kpc}$ and a smoother trend between 2 and $6.4 \mathrm{Kpc}$; both features are much more pronounced in the $B$ than in the infrared band. This can be due to the presence of bursts of star formation induced by the interaction (Toomre 1972). Nevertheless, it has been possible to fit the disk by using Eq. (1); the calculated values for the radial scalelengths, in Kpc, are given in Table 8 , with those for the bulge in all five photometric bands, even if the fits are only indicative of the presence of bulge 
and disk components and not a real approximation of the measured profiles, due to the profile bumps (see Fig. 16).

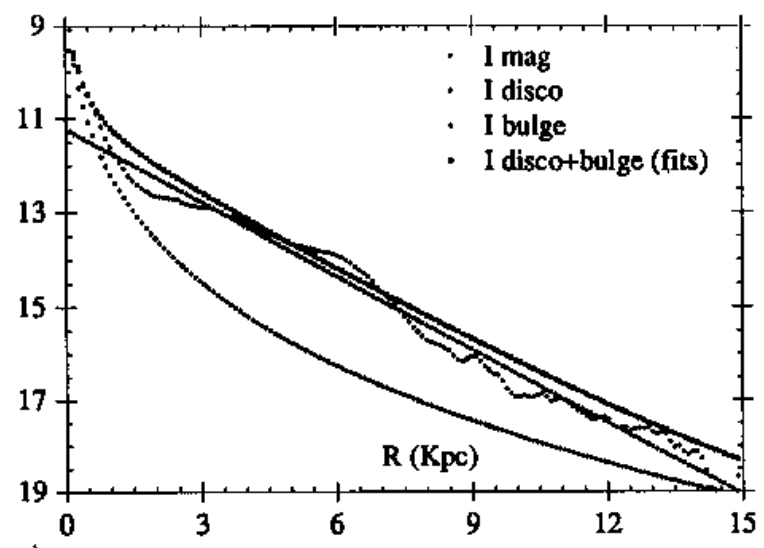

Fig. 16. The $I$ band surface brightness radial profile for NGC 5194 and, superimposed, the disk fit, the bulge one and the composite trend disk + bulge. The disk fit formula is given by Eq. (1), see the text

Table 8. $r_{\mathrm{d}}, r_{\mathrm{e}}, D / B$ for NGC 5194

\begin{tabular}{lllll}
\hline & $U$ & $B$ & $V$ & $I$ \\
\hline$r_{\mathrm{d}}(1)$ & 2.07 & 1.92 & 1.95 & 2.08 \\
$r_{\mathrm{e}}$ & 1.67 & 1.17 & 1.09 & 1.85 \\
$D / B(1+3)$ & 4.37 & 7.53 & 3.63 & 2.74 \\
\hline
\end{tabular}

As can be seen from Table 8 , the $D / B$ parameter decreases toward longer wavelengths, with the exception of the $U$ band, which confirms on the one hand the presence of a huge amount of young stars in the disk, and on the other the interaction-induced starburst hypothesis. Disk scalelengths, on the contrary, are more or less constant with various wavelengths. At $10 \mathrm{Kpc}$, another sudden change in the slope of the disk profile, again due to the companion takes place. A full comparison between our data and those from Boroson (1981), is not possible, because Boroson's profiles are measured only up to $5-$ $6 \mathrm{Kpc}$; however, in this range, there is a good agreement between his profiles and ours. The colour indexes are given in Figs. 17b; note in $(B-V)$ and $(B-I)$ a reddening in correspondence with the slope change in the disk zone and a specular bluish in the same zone for the $(U-B)$ index.

It is possible that the red peak in $(B-V)$ and in $(B-I)$ is due to a dust concentration (Prieto et al. 1992) originated from the tidal interaction; this may be confirmed by

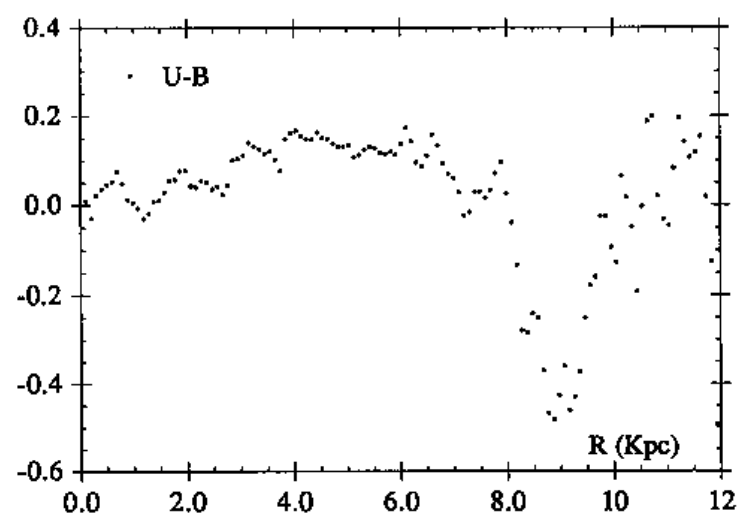

Fig. 17. a) Radial trend of the colour indexes $(B-I),(B-V)$ with galactocentric radius

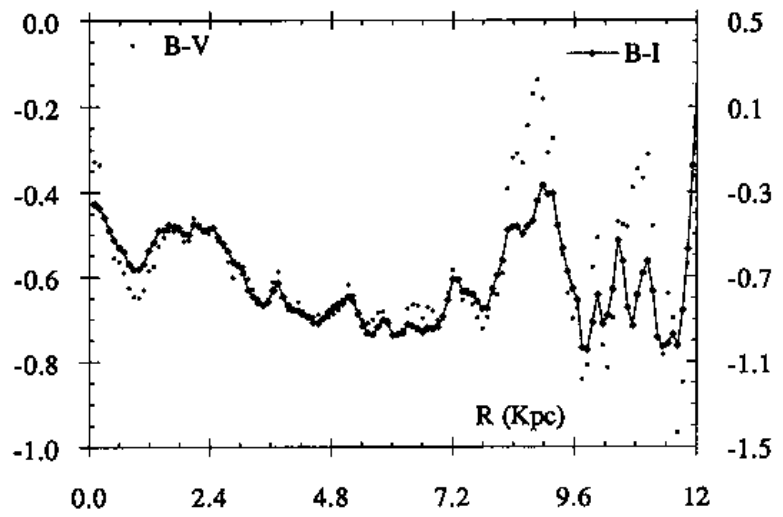

Fig. 17. b) Radial trend of the colour indexes $(U-B)$ with galactocentric radius

a second, less pronounced peak between 1.5 and $3 \mathrm{Kpc}$. For small radii both $(B-V)$ and $(B-I)$ are bluish; it is not clear if this fact is due to the perturbation (infall) or to an inner spiral arm. There is also a $1: 2$ scaling ratio between the $(B-V)$ and $(B-I)$ indexes.

\section{Summary and discussion}

It can be seen from the the section above that four galaxies in our sample have a knee in the radial disk profile, NGC 6946, NGC 3938, NGC 3184, NGC 3423, and two of them have also a Freeman II profile, NGC 3184, NGC 3423. For these two galaxies, we have tried to fit the disk trend using Eq. (2), $m(r)=$ $m(0)+1.0857\left(\alpha r-\left(\frac{\beta}{r}\right)^{n}\right)$, with $\alpha=\beta$, and $n=3$. For the other two galaxies, we have noticed that a single exponential fit is not adequate for the whole disk, and that the calculated values for the radial scalelengths vary with the radial range considered, as well as the central disk surface brightness. If only the disk is considered, these results 
seem to agree with the prediction of Phillips et al. (DDP model). In their paper these authors present a model of dust distribution, called "Triplex model", with the following assumptions:

a) the galaxy is made by a pure exponential disk (the bulge component is not considered),

b) stars and gas are exponentially distributed both radially and vertically,

c) the radial scalelengths of dust and stars are equal, while the dust scaleheight is smaller than that of stars.

The physical consequences of this model are threefold, (Davies 1990):

1) galactic disks are optically thick till $22-23 \mathrm{a}$ mag $B$;

2) the exponential behaviour of dust and gas implies that the measurements of the bulge mass from $M / L$ ratios are wrong, because of the attenuation of luminosity by dust. The trend of inner rotation curves measured in spiral galaxies can be explained as a consequence of a more massive bulge than that estimated by optical measurements;

3) the infrared to optical luminosity ratio must be quite large $\left(10^{2}\right)$, because light is absorbed in the optical region and is re-emitted in the infrared (this value has yet been found by IRAS for nearby galaxies, see Davies 1990 and references therein).

In this view the difference between Freeman I and Freeman II profiles is explained in terms of a dimming in disk luminosity, caused by dust and by a strong emission from a massive bulge that compensates for this dimming (Freeman I), or from a little bulge, unable to compensate for the dimming, so that the radial surface brightness profile shows a drop in the bulge-disk transition zone (Freeman II). The observed profiles, however, disagree with model predictions when the bulges are taken into account, and a total fit bulge + disk is superimposed to data, as it can be seen from Figs. 5 and 7. We have also noticed that radial disk scalelengths vary with wavelength; in fact it is larger in blue than in infrared for NGC 1232, NGC 6946, while the opposite trend takes place for the other galaxies. The first result agrees with the predictions of the DDP model, but it is also in good agreement with the interpretation of RD. However it can be seen from the tables that a $r_{\mathrm{d}}$ trend with $\lambda$ similar to the RD one is observed only for two peculiar galaxies, and that the smoothest increase of the radial disk scalelength with the wavelength is found for two other peculiar galaxies, NGC 4321 and NGC 5194, while a pronounced increase of $r_{\mathrm{d}}$ with $\lambda$ is observed for NGC 3184, NGC 3423, NGC 3938. This result for NGC 3423 is in disagreement with that found by RD. The radial profile of the color index $(B-V)$ of NGC 3184 shows a trend toward blue for small values of radius; this has been interpreted by Larson \& Tinsley (L\&T78) as an infall of gas toward the galactic nuclei. As this phenomenon is not present in all galaxies, they assume that a galactic wind blows up the infall in some cases. When this occurs, the $(B-V)$ index is red toward the galaxy centre, i.e. like the one of NGC 1232. Another interpretation is given by $\mathrm{RD}$; according to these authors there is a radial inflow of gas toward the galaxy centre, driven by a bar. This inflow can also be responsible for the Freeman II profile. Radial gradients have been found in the colour indexes $(B-I)$ and $(B-V)$; according to the Larson model (Larson 1976), these are due to a decrease in age and metallicity toward the edge of galaxy. A peculiar behaviour is observed in the colour indexes $(B-I)$ and $(B-V)$ of NGC 4321 (M 100) and of NGC 5194 (M 51); they show a strong red peak corresponding to a drop in luminosity, which occurs in the radial surface brightness profiles, in particular in the $B$ one. This reddening, corresponding to the dimming in luminosity profiles, confirms, according to Prieto, the presence of a huge amount of dust in the bulge to disk transition zone. It has not been possible to apply the same consideration to the same zone in NGC 3184, where the colour index is flat in correspondence of the flat zone in the luminosity profile. The two peculiar galaxies, M 51 and NGC 4321, in particular M 51, show a real irregular luminosity profile, so the decomposition into bulge and disk components is less reliable than that obtained for the other galaxies. There is a $1: 2$ scaling ratio between the $(B-V)$ and the $(B-I)$ index for all the galaxies in our sample. We also want to point out that our choice for the fitting formulas for bulge and disk is not unique; in the work by Vila Costas \& Edmunds (1992) the definition of the disk scalelengths is given as that radius at which the disk luminosity is one half of its central value. This means that Eq. (1) must be rewritten as: $m(r)=m\left(r_{\mathrm{d}}\right)+1.822\left(\frac{r}{r_{\mathrm{d}}}\right)$. If one does not specify which fit is used in the work, some misunderstanding can arise, and there is the possibility to find values for the disk scalelengths which differ, from author to author, by a factor of about two. This phenomenon has already been considered in a work by Knapen \& Van der Kruit (1991). These authors, starting from these discrepancies, question about the usefulness of the bulge to disk decomposition and its physical meaning, but, perhaps all the differences can be explained in term of a different choice of the fitting formula.

Acknowledgements. The authors wish to thank Dr. F. Pedichini for his precious help in the acquisition of data, Dr. Francesca Natali for the helpful discussion during the data reduction, Dr. P. Monaco for reading the first version of the paper and A. Di Clemente for his help during the observations

\section{References}

Boroson T., 1981, ApJS 46, 177

Davies I.J., 1990, MNRAS 245, 350

De Vaucouleurs G., 1974, in: Structure, Dynamics and Statistical Properties of the Galaxies, Reidel J.R. (eds.) p. 1 
Disney M., Davies J.I., Phillips S., 1989, MNRAS 239, 939 (DDP model)

Freeman K.C., 1970, ApJ 160, 811

Holmberg E., 1958, Med. Lunds Astr. Obs., Ser. 2, No. 36

Kent S.M., 1985, ApJS 59, 115

Knapen J.H., Van der Kruit P, 1991, A\&A 248, 57

Kormendy J., 1977, ApJ 217, 406

Larson R.B., 1976, MNRAS 176, 31

Larson R.B., Tinsley B.M., 1978, ApJ 219, 46

Natali G., Pedichini F., Righini M., 1992, A\&A 256, 79 (Paper I)
Phillips S., Evans R.H., Davies I.J., Disney M., 1989, MNRAS 253, 496

Prieto M., Beckman J.E., Varela A.M., 1992, A\&A 257, 85

Ryder S.D., Dopita M.A., 1994, ApJ 430, 142 (RD)

Toomre A., Toomre J., 1972, ApJ 178, 623

Tully R.B., 1980, Nearby Galaxies Catalog. Cambridge University Press

Van der Bergh S., 1976, ApJ 206, 883

Vila Costas M.B., Edmunds M.G., 1992, MNRAS 259, 121

Young J.S., Scoville N., 1982, ApJ 258, 467 